Allgemeine Betriebswirtschaftslehre in programmierter Form 


\section{Allgemeine \\ Betriebswirtschaftslehre \\ in programmierter Form}

Herausgeber: Prof. Dr. H. Jacob

unter Mitarbeit von:

(in alphabetischer Reihenfolge)

Prof. Dr. W. Busse von Colbe, Universität Bochum

Prof. Dr. H. Diederich, Universität Mainz

Prof. Dr. Dr. h. c. E. Heinen, Universität München

Prof. Dr. W. Kern, Universität Köln

Prof. Dr. W. Kilger, Universität Saarbrücken

Prof. Dr. H. Kossbiel, Universität Hamburg

Prof. Dr. E. Witte, Universität München

Dritte, neu bearbeitete und erweiterte Auflage 
Additional material to this book can be downloaded from http://extras.springer.com ISBN 978-3-663-00063-1 DOI 10.1007/978-3-663-00213-0

Copyright by Springer Fachmedien Wiesbaden 1976 Ursprünglich erschienen bei Dr. Th. Gabler-Verlag, Wiesbaden 1976

Softcover reprint of the hardcover 1st edition 1976 


\section{Vorwort des Herausgebers zur ersten Auflage}

Das vorliegende Buch enthält - in der Form von Frage und Antwort, Aufgabe und Lösung - eine Darstellung des Stoffes, der heute als gesichertes Wissen auf dem Gebiet der Allgemeinen Betriebswirtschaftslehre vorliegt.

Die im Untertitel angezeigte vom Ublichen abweichende Art der Darstellung wurde gewählt, um diesen umfangreichen Stoff in kleinen Schritten, straff gegliedert und jeweils das Wesentliche herausstellend, dem Leser darbieten zu können. Mit jeder Frage wird ein bestimmtes Problem angesprochen. Die Fragen sind dergestalt miteinander verknüpft, daß sie das jeweilige Gebiet Schritt für Schritt in systematischer Abfolge erfassen. Es soll dadurch erreicht werden, daß die Gesamtzusammenhänge trotz der Aufteilung des Stoffes in "Lerneinheiten" nicht verwischt werden, sondern deutlich erkennbar bleiben.

Für den Lernenden bedeutet diese Art der Darstellung:

- Er empfängt in systematischer Folge Anstöße, die ihn zu einem aktiven Verhalten, zum Mitdenken und Mitarbeiten anregen;

- er wird in die Lage versetzt, seinen jeweiligen Wissensstand selbst zu überprüfen.

Ein "Lernprogramm" besteht im allgemeinen aus der Darlegung des die "Lerneinheit" bildenden Stoffes. Am SchluB jeder Lerneinheit steht eine Frage mit richtiger Antwort, die es dem Lernenden ermöglichen soll festzustellen, ob oder auch inwieweit er sich den behandelnden Stoff aneignen konnte. Von dieser Reihenfolge ist hier insoweit abgewichen, als zunächst die Frage gestellt und damit ein bestimmtes Problem angesprochen wird. Die Antwort ist alsdann so ausführlich gehalten, daß auch derjenige, dem bislang der durch die Fragen angesprochene Problemkreis wenig oder nicht bekannt war, nun umfassend damit vertraut gemacht wird. Dieses Vorgehen erschien zweckmäßig, da es in noch stärkerem Maße zu selbständigem Denken und eigener Stellungnahme herausfordert.

Durch die eingestreuten Aufgaben soll die Anwendung bestimmter Methoden und Techniken dort, wo es notwendig erscheint, geübt und die Anwendung des Gelernten auf konkrete Fälle ermöglicht werden. Solche Aufgaben finden sich z. B. in den Abschnitten Preispolitik, Werbung. Finanzierung, Investitionsrechnung, Bilanzen und Kostenrechnung. 
Um das Buch lesen zu können, bedarf es keiner besonderen betriebswirtschaftlichen Vorkenntnisse. Die Fragen und Antworten sind so formuliert und angeordnet, daß sie auch für Studierende der ersten Semester verständlich sind und geeignet erscheinen, in die Betriebswirtschaftslehre einzuführen und mit den Fragestellungen, dem Aufbau und den Methoden dieses Wissengebietes vertraut zu machen.

Der Gesamtstoff ist in acht Kapitel gegliedert, die von verschiedenen Autoren, jedoch in gleicher Form und mit der gleichen Zielsetzung geschrieben wurden. Nach einer Darstellung des Erkenntnisobjektes, der Methoden und einiger Grundbegriffe der Betriebswirtschaftslehre, ferner nach einem Uberblick über die rechtlichen Gestaltungsmöglichkeiten (Rechtsformen, Betriebszusammenschlüsse) im ersten Kapitel, befaßt sich das zweite Kapitel mit den Elementen, dem Aufbau und der inneren Struktur der Betriebe. Auch Fragen der Planung und Organisation, der Kräfte also, die aus den elementaren Faktoren ein sinnvolles Ganzes aufbaven, werden hier behandelt. Die Kapitel III, IV und V sind den großen betrieblichen Teilbereichen der Leistungserstellung (Produktions- und Kostentheorie), der Leistungsverwertung (Absatz einschließlich Preispolitik) und der Finanzwirtschaft der Unternehmung gewidmet. Die Methoden der Investitionsrechnung und ihre Anwendung ebenso wie die Bewertung einer Unternehmung im ganzen bilden den Inhalt des sechsten Kapitels. Die beiden letzten Kapitel schließlich befassen sich mit dem betrieblichen Rechnungswesen. In Kapitel VII wird das große Gebiet der Bilanzierung einschließlich Kapitalflußrechnung, Bilanzanalyse und Bilanztheorie abgehandelt, während in Kapitel VIII die Grundlagen und Formen der Kostenrechnung sowie die Planung und Kontrolle des Periodenerfolges dargestellt sind. 


\section{Vorwort des Herausgebers zur dritten Auflage}

Während die zweite Auflage im wesentlichen einen um Druckfehler u. ä. berichtigten Nachdruck der ersten darstellte, wurde bei der hier vorliegenden dritten Auflage die Gelegenheit wahrgenommen, den gesamten Text gründlich zu überarbeiten und notwendig erscheinende - zum Teil recht umfangreiche - Ergänzungen vorzunehmen. Daß dadurch die Seitenzahl um über $20 \%$ anstieg, erwies sich als unumgänglich. Nur so war es möglich, eine dem heutigen Stand des Wissens entsprechende, in sich geschlossene, umfassende Darstellung des Stoffes der Allgemeinen Betriebswirtschaftslehre zu bieten.

Zu den bisher acht Kapiteln ist ein neuntes hinzugekommen: Die rasche Entwicklung auf dem Gebiet der Personalwirtschaftslehre ließ es erforderlich erscheinen, die Teile dieser Lehre, die für alle Betriebe bedeutsam sind, also zur Allgemeinen Betriebswirtschaftslehre gehören, in einem zusätzlichen neunten Kapitel darzustellen.

Von den zahlreichen Ergänzungen und Erweiterungen, die in den Kapiteln 1 bis 8 vorgenommen wurden, können hier nur einige genannt werden: Auf dem Gebiet der Produktions- und Kostentheorie zeichneten sich in den letzten Jahren vor allem zwei Entwicklungstendenzen ab: Zum einen verstärkten sich die Bestrebungen, die Produktions- und Kostentheorie mit einer Theorie der Produktionsplanung zu verbinden. Impulse hierzu gingen nicht zuletzt auch von der Entwicklung praxisrelevanter, computergestützter Konzeptionen zur Fertigungssteuerung aus. Zum andern war man bemüht, dem Faktor Arbeit einen besonderen Stellenwert einzuräumen und die bisher vorrangig technisch-mechanistisch ausgerichtete Produktions- und Kostentheorie um diesen Aspekt zu erweitern. In dem Kapitel "Produktions- und Kostentheorie" wurde diesen Entwicklungen Rechnung getragen.

Die Darstellung never Finanzierungsformen und international never Denkansätze zur Optimierung der Finanzstruktur vervollständigen die Ausführungen zur Finanzwirtschaft der Unternehmung. Ferner erschien es hier geboten, auf moderne Prognoseverfahren zur Ermittlung des Kapitalbedarfs und der künftigen finanziellen Entwicklung einzugehen.

Das Kapitel "Investitionsrechnung" umfaßt nun auch eine ausführliche Beschreibung der Investitionsmodelle, die auf der linearen Programmierungsrechnung basieren. Beginnend mit den einfachen, einperiodigen Kapitalwertmodellen, wird die Entwicklung bis hin zu den mehrperiodigen Integrationsmodellen nachgezeichnet und erläutert, wobei auch die Anwendungsmöglichkeiten der einzelnen Modellformen erklärt werden. 
Das Kapitel „Bilanzen" wurde vor allem im Hinblick auf die inzwischen eingetretenen Anderungen handels- und steuerrechtlicher Vorschriften aktualisiert. Im Abschnitt "Bilanztheorie" ist zusätzlich zu dem heute wieder aktuellen Problem der Eliminierung von Preisschwankungen und zu dem in der jüngeren Literatur ausgiebig diskutierten Konzept des ökonomischen Gewinns Stellung genommen.

Dem Kapitel "Betriebliches Rechnungswesen" wurde ein neuer Abschnitt über die Entwicklungsformen der Kostenrechnung angefügt. Ausgebaut wurde hier auch der Abschnitt über die Grenzplankosten- und Deckungsbeitragsrechnung.

Für die Darstellung und Aufbereitung des Lehrstoffes ebenso wie für die Zielsetzung des Buches trifft das bereits im Vorwort zur ersten Auflage Gesagte unverändert zu.

Ich danke allen, die durch ihre Mitwirkung und Unterstützung diese dritte Auflage ermöglicht haben. Dieser Dank gilt insbesondere auch den Mitarbeitern des Verlages für manche wertvolle Anregung und eine stets gute Zusammenarbeit. 


\section{Inhaltsverzeichnis}

\section{Grundtatbestände der Betriebswirtschaftslehre}

von Prof. Dr. Helmut Diederich, Mainz . . . . . . . . . . . . . . 17

I. Grundtatbestände und Grundbegriffe . . . . . . . . . . . . . 21

1. Der Betrieb als Gegenstand der Betriebswirtschaftslehre . . . . . 21

a) Wesen und Aufgaben der Betriebe . . . . . . . . . . 21

b) Betriebstypen ................. . . 26

2. Die Betriebswirtschaftslehre ............ 28

a) Erkenntnisziel der Betriebswirtschaftslehre . . . . . . . 28

b) Gliederung der Betriebswirtschaftslehre . . . . . . . . . . 55

c) Abgrenzung zu Nachbardisziplinen . . . . . . . . . . . 57

d) Geschichte der Betriebswirtschaftslehre ........... 60

3. Wirtschaftlichkeit, Produktivität und Rentabilität als Kennzahlen

betrieblicher Betätigung ............ 63

II. Methoden betriebswirtschaftlicher Forschung . . . . . . . . . 73

1. Aufgaben der Methodologie . . . . . . . . . . 73

2. Die in der Betriebswirtschaftslehre verwendeten Methoden . . . . 75

III. Die Rechtsformen der Betriebe . . . . . . . . . . . . . . . . 81

1. Oberblick über die Rechtsformen . . . . . . . . . . 81

2. Die Bestimmung der jeweils optimalen Rechtsform . . . . . . . . 84

3. Rechtsformen begrenzter Anwendbarkeit .......... 90

IV. Betriebliche Zusammenschlüsse . . . . . . . . . . . . 96

1. Die Kooperation . . . . . . . . . . . . . 96

2. Zusammenschlüsse unter einheitlicher Leitung . . . . . . . . 105

Literaturverzeichnis . . . . . . . . . . . . 111

Stichwortverzeichnis . . . . . . . . . . . . 115 


\section{Der Betrieb als Faktorkombination}

von Prof. Dr. Werner Kern, Köln . . . . . . . . . . . . . . . . . . . . 117

A. Systematisierung der Produktionsfaktoren . . . . . . . . . . . . 121

B. Die originären Produktionsfaktoren . . . . . . . . . . . 125

I. Der Produktionsfaktor "menschliche Arbeit" . . . . . . . . 125

II. Der Produktionsfaktor "Betriebsmittel" . . . . . . . . . . . . 132

III. Der Produktionsfaktor "Werkstoffe" . . . . . . . . . . . . 138

IV. Der Produktionsfaktor "Betriebs- und Geschäftsleitung" . . . . . . 140

C. Der derivative Produktionsfaktor "Planung" (und sein auf die Faktorkombination ausgerichtetes Wesen) ........... 145

I. Grundlagen . . . . . . . . . . . . . . . . . . 145

II. Der Planungsprozeß . . . . . . . . . . . . . . . . . 155

III. Planung einzelner betrieblicher Funktionen . . . . . . . . . 168

D. Der derivative Produktionsfaktor "Organisation" (und sein auf die Faktorkombination ausgerichtetes Wesen) . . . . . . 176

I. Grundlagen . . . . . . . . . . . . . . 176

II. Aufbauorganisation (Gestaltung des Potentialgefüges) . . . . . . 183

III. Ablauforganisation (Gestaltung des Aktionsgefüges) . . . . . . 192

Literaturverzeichnis . . . . . . . . . . . . . . . 201

Stichwortverzeichnis . . . . . . . . . . . . . . 203

\section{Produktions- und Kostentheorie}

von Prof. Dr. Dr. h. c. Edmund Heinen, München . . . . . . . . . . . . . 205

I. Gegenstand, Aufbau und Aufgaben der betriebswirtschaftlichen Kostenlehre . . . . . . . . . . . . . . . . . . . 209

II. Die Produktionsfunktion als produktions- und kostentheoretisches Grundmodell . . . . . . . . . . . . . . . . . . 221

1. Allgemeine Grundlagen . . . . . . . . . . . . . . 221

2. Die Produktionsfunktion vom $\operatorname{Typ} A \ldots \ldots 223$

3. Die Produktionsfunktion vom Typ B . . . . . . . . . . 230

4. Die Produktionsfunktion vom $\operatorname{Typ} C \ldots \ldots . \ldots 236$

5. Die Berücksichtigung der menschlichen Arbeit . . . . . . . . . 254 
III. Instrumente und Formen kostentheoretischer Modellanalyse . . . . 256

IV. Die Theorie der Kosteneinflußgrößen . . . . . . . . 266

V. Kostentheoretische Modellansätze und organisatorischer

Entscheidungsproze $\beta \ldots \ldots$. . . . . . . . . 285

Literaturverzeichnis . . . . . . . . . . . . . . . . 291

Stichwortverzeichnis . . . . . . . . . . . . . 293

\section{Der Absatz}

von Prof. Dr. Herbert Jacob, Hamburg . . . . . . . . . . . . . . . 297

A. Die Aufgaben der Unternehmung im Absatzbereich . . . . . . . 301

B. Die Absatzplanung . . . . . . . . . . . 306

I. Die Daten. . . . . . . . . . . . . . . 306

1. Gesamtwirtschaftliche Größen. Indikatoren . . . . . . . 306

2. Instrumentalinformationen . . . . . . . . . . 314

II. Die Gewinnung der Daten: die Marktforschung . . . . . . . . . . 317

1. Die Forschungsgebiete . . . . . . . . . . 318

2. Die Verfahren der Marktforschung . . . . . . . 322

III. Der Absatzplan . . . . . . . . . . . . . 331

C. Produktstrategie $\ldots \ldots \ldots \ldots \ldots \ldots$

I. Produktvariation . . . . . . . . . . . . . . . 341

1. Arten und Möglichkeiten . . . . . . . . . . . . 341

2. Die Marktaufspaltung (Marktsegmentation) . . . . . . . 347

II. Die Produktlinie . . . . . . . . . . . . . . . 353

III. Die Einführung never Produkte. Produktdiversifikation . . . . . . 358

D. Preispolitik . . . . . . . . . . . . . . 364

I. Grundbegriffe. Daten der Preispolitik. Formen der Preisbildung . . . 364

II. Die Preispolitik monopolistischer Anbieter und Nachfrager . . . . . 369

1. Die Darstellung der Absatzsituation . . . . . . . . . 369

2. Die Ableitung des Gewinnmaximums (der Cournotsche Punkt) . . 372 
3. Preisstellung bei anderen Zielsetzungen . . . . . . . 375

4. Preisstellung nach Maßgabe der Durchschnittskosten und der Grenzkosten . . . . . . . . . . . . . . . . 378

5. Das Nachfragemonopol . . . . . . . . . . . . . . . 382

III. Preispolitik im Polypol . . . . . . . . . . . . . . . . . 383

1. Polypolistische Konkurrenz auf vollkommenen Märkten . . . . . 384

2. Polypolistische Konkurrenz auf unvollkommenen Märkten . . . . 390

IV. Preispolitik auf Gebietsmärkten, Preisdifferenzierung, Sonderfragen . 397

1. Preispolitik auf Gebietsmärkten . . . . . . . . . . . . . 397

2. Preisdifferenzierung . . . . . . . . . . . 407

3. Preisstellung bei Kuppelproduktion . . . . . . . . . . 413

4. Preisstellung bei absatzmäßiger Verflechtung der Erzeugnisse . . 415

5. Rabatte, Zusatzaufträge . . . . . . . . . . . . 417

V. Preispolitik im Dyopol und Oligopol . . . . . . . . . . . 418

A. Dyopol und Oligopol auf unvollkommenen Märkten . . . . . 419

1. Wirtschaftsfriedliches Verhalten . . . . . . . . . 419

a) Preisstellung bei voller Kenntnis der Daten . . . . . . . . 419

b) Preisstellung auf der Grundlage von Reaktionserwartungen Der tabellarische Lösungsansatz . . . . . . . . 431

2. Kollektive Preispolitik . . . . . . . . . . . 434

3. Kampfsituationen . . . . . . . . . . . . . 437

B. Dyopol und Oligopol auf vollkommenen Märkten . . . . . . 438

VI. Das bilaterale Monopol . . . . . . . . . . . . . . . 440

VII. Die vertikale Preisbindung . . . . . . . . . . . . 447

E. Die Absatzmethoden . . . . . . . . . . . . . . . 440

I. Die Vertriebssysteme . . . . . . . . . . . . . . 451

II. Die Absatzformen . . . . . . . . . . . . . . 452

III. Die Absatzwege . . . . . . . . . . . . . . . . . 459

IV. Das Wahlproblem im Hinblick auf die Absatzmethoden,
insbesondere die Absatzwege und Absatzgebiete . . . . . 468

V. Gestaltungsmöglichkeiten im Export . . . . . . . . . . . . 473 
F. Die Werbung . . . . . . . . . . . . . . 4474

I. Begriffliches, Abgrenzungen, Ziele . . . . . . . . . 474

II. Die Entscheidungsgebiete im Rahmen der Werbeplanung . . . . . 476

III. Zur Theorie der Werbung . . . . . . . . . . . . . . 483

1. Die Grundkonzeption . . . . . . . . . . . . . . 483

2. Werbekosten und Absatz . . . . . . . . . . 485

3. Die Bestimmung des optimalen Werbebudgets (Einperiodenbetrachtung) . . . . . . . . . . . 491

a) Beim Absatz eines Erzeugnisses . . . . . . . . . . . . . 491

b) Beim Absatz mehrerer Erzeugnisse . . . . . . . . . . 495

4. Die Mehrperiodenbetrachtung . . . . . . . . . . . . 497

IV. Möglichkeiten zur Sicherung des Werbeerfolgs.

Die Werbeerfolgskontrolle . . . . . . . . . . . 498

Literaturverzeichnis . . . . . . . . . . . . . 505

Stichwortverzeichnis . . . . . . . . . . . . . 511

\section{Die Finanzwirtschaft der Unternehmung}

von Prof. Dr. Eberhard Witte, München . . . . . . . . . . . . . 515

I. Der Problemkomplex der Finanzwirtschaft . . . . . . . . . 519

1. Begriff der Finanzwirtschaft ............ . . 51s

2. Finanzwirtschaftliche und erfolgswirtschaftliche Betrachtungsweise. . 520

3. Liquidität und Gewinn . . . . . . . . . . . . . 522

II. Kapitalbedarf und Finanzprognose . . . . . . . . . . . . . 528

1. Kapitalbedarf . . . . . . . . . . . . . 528

2. Kapitalbindungsplan ................. 529

3. Finanzplan .................... 533

4. Täglicher Finanzstatus . . . . . . . . . . . 543 
III. Finanzierungsalternativen . . . . . . . . . . . . . . 545

1. Finanzierungssystematik . . . . . . . . . . . . 545

2. Fremdfinanzierung . . . . . . . . . . . . 547

a) Lieferantenkredit . . . . . . . . . . . . . . . 550

b) Kundenanzahlung . . . . . . . . . . . . . . . . . . 551

c) Factoring . . . . . . . . . . . . . . . . . . 551

d) Wechselkredit . . . . . . . . . . . . . . . . 552

e) Kontokorrent-Kredit . . . . . . . . . . . . . . . . 555

f) Darlehen . . . . . . . . . . . . . . . . . . 559

g) Obligation . . . . . . . . . . . . . . . . . . 562

h) Leasing . . . . . . . . . . . . . . . . . . 564

i) Langfristige Rückstellungen . . . . . . . . . . . . . . . . 566

j) Verzögerte Gewinnsteverabführung . . . . . . . . . . . . 566

3. Eigenfinanzierung . . . . . . . . . . . . 567

4. Selbstfinanzierung . . . . . . . . . . . . 572

5. Finanzierung aus Desinvestitionen . . . . . . . . . 575

IV. Finanzierungsentscheidung ． . . . . . . . . . . . 577

1. Kriterien der Finanzierungsentscheidung . . . . . . . . . 577

a) Sicherheit . . . . . . . . . . . . . . . . . . 577

b) Wirtschaftlichkeit . . . . . . . . . . . . . . . 582

c) Autonomie . . . . . . . . . . . . . . . . . . 589

2. Die ,ideale Finanzierungsstruktur" . . . . . . . . . . . 591

V. Finanzwirtschaftliche Anpassungsentscheidungen bei begrenzter Finanzierung . . . . . . . . . . . . . . 592

1. Grenzen der Finanzierung . . . . . . . . . . . . 592

2. Liquiditätssicherung bei begrenzter Finanzierung . . . . . . . . 594

a) Liquiditätsengpaß . . . . . . . . . . . . . . . 594

b) Maßnahmen zur Oberwindung eines Liquiditätsengpasses . . . . 595

c) Entscheidung über liquiditätspolitische Maßnahmen . . . . . . 599

3. Position des Finanzleiters im Liquiditätsengpaß . . . . . . . 600

Literaturverzeichnis . . . . . . . . . . . . . 603

Stichwortverzeichnis . . . . . . . . . . . . . 6 607 


\section{Investitionsrechnung}

von Prof. Dr. Herbert Jacob, Hamburg . . . . . . . . . . . . . . . . . . 609

I. Aufgaben, Grundlagen und Grundbegriffe der Investitionsrechnung . . . 615

II. Kriterien zur Beurteilung einer einzelnen Investition . . . . . . . . . . 619

1. Die Kapitalwertmethode . . . . . . . . . . . . . . . . 619

2. Die Methode des internen Zinsfußes . . . . . . . . . . . . 621

3. Die Annuitätsmethode . . . . . . . . . . . . 623

4. Die Ermittlung von Pay-off-Zeiten . . . . . . . . . . . . 628

5. Der Kalkulationszinsfuß . . . . . . . . . . . 632

6. Sonderfragen .................... 6634

a) Der Einfluß der Besteuerung . . . . . . . . . . . . 634

b) Die Unsicherheit der Daten . . . . . . . . . . . . . . . 635

III. Das Wahlproblem . . . . . . . . . . . . . 636

IV. "Kritische Werte” und ihre Bedetung für Investitionsentscheidungen . . . 641

V. Die Bestimmung der optimalen Nutzungsdauer einer Anlage und das Ersatzproblem . . . . . . . . . . . . . . 647

1. Die Bestimmung der optimalen Nutzungsdauer einer nicht zu ersetzenden Anlage . . . . . . . . . . . . 647

2. Das Ersatzproblem . . . . . . . . . . . . 652

a) Steigende Betriebsausgaben . . . . . . . . . . . . . 652

b) Auftreten einer funktionsgleichen, kostengünstigeren Anlage . . . 655

c) Ersatzvergleich und Umverteilungseffekt . . . . . . . . . . 662

d) Berücksichtigung eines zukünftigen technischen Fortschritts . . . 667

VI. Die Ermittlung des Investitionsprogramms . . . . . . . . . . 672

VII. Investitionsmodelle auf der Grundlage der linearen

Optimierungsrechnung

1. Kapitalwertmodelle . . . . . . . . . . . . 683

2. Integrationsmodelle .............. . . 693 
VIII. Die Bewertung ganzer Unternehmungen . . . . . . . . . . . 703

IX. Anhang: Zinseszinsfaktoren . . . . . . . . . 716

Literaturverzeichnis . . . . . . . . . . . . . . 717

Stichwortverzeichnis . . . . . . . . . . . . . . 719

\section{Bilanzen}

von Prof. Dr. Walther Busse von Colbe, Bochum . . . . . . . . . . . 721

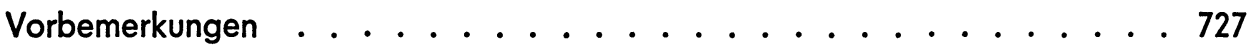

I. KapitalfluBrechnungen . . . . . . . . . . . . 729

1. Aufstellung von Kapitalflußrechnungen aus Geschäftsvorfällen nach verschiedenen Fondskonzepten . . . . . . . . . . 729

2. Aufstellung von Bewegungsbilanzen . . . . . . . . 733

3. Vergleich der Aussagefähigkeit von Kapitalflußrechnungen und Bewegungsbilanzen ............. 735

4. Aufstellung von Kapitalflußrechnungen und Bewegungsbilanzen aus dem aktienrechtlichen Jahresabschluß . . . . . . . . 737

II. Bilanzierungspflicht und Bilanzierungsgrundsätze . . . . . . 742

1. Pflicht zur Bilanzaufstellung . . . . . . . . . . . . 742

2. Grundsätze ordnungsmäßiger Bilanzierung . . . . . . . 745

3. Feststellung und Publizität des Jahresabschlusses . . . . . . 752

III. Bilanzierung des Anlagevermögens . . . . . . . . . . 756

1. Bewertung und Abschreibung von Sachanlagen . . . . . . 756

2. Bilanzierung immaterieller Anlagewerte ... . . . . . 769

3. Bilanzierung von Finanzanlagen . . . . . . . . . 771

IV. Bilanzierung des Umlaufvermögens . . . . . . . . . . . . . 777

1. Inventur . . . . . . . . . . . . . . 777

2. Bestandteile der Anschaffungs- und Herstellungskosten . . . . 780

3. Bewertungsverfahren für Vorräte . . . . . . . . . . 788 
2. Die Produktionsfaktoren im System des betrieblichen Rechnungswesens . . 905 21. Die Materialabrechnung .. . . . . . . . . . . . . . 905

22. Die Lohnabrechnung . . . . . . . . . . . . . . . . . . . 908

23. Die Betriebsmittelabrechnung .. . . . . . . . . . . . 911

3. Die Entwicklungsformen der Kostenrechnung . . . . . . . . . . . . . 921

31. Die Istkostenrechnung . . . . . . . . . . . . . . . . 921

32. Die Normalkostenrechnung . . . . . . . . . . . . . . . 922

33. Die Plankostenrechnung . . . . . . . . . . . . 925

4. Funktionsbereiche der Kostenrechnung . . . . . . . . . . . . . . . 930

41. Die Kostenartenrechnung . . . . . . . . . . . . . . . . . 930

42. Die Kostenstellenrechnung . . . . . . . . . . . . . . . 932

43. Die Kostenträgerstückrechnung . . . . . . . . . . . . . . 945

5. Die Grenzplankostenrechnung und Deckungsbeitragsrechnung . . . . . 972

51. Der Aufbau einer Grenzplankostenrechnung . . . . . . . . . . . 972

52. Die Durchführung einer Grenzplankostenrechnung . . . . . . . . . 975

6. Die kurzfristige Erfolgsrechnung (Kostenträgerzeitrechnung ) . . . . . . . 980

61. Verfahren der kurzfristigen Erfolgsrechnung . . . . . . . . . . 980

62. Die Erfolgsanalyse im System der Grenzplankostenrechnung . . . . 986

63. Die kurzfristige Erfolgsrechnung als unternehmerische Dispositionshilfe 995

Literaturverzeichnis . . . . . . . . . . . . . . 1003

Stichwortverzeichnis . . . . . . . . . . . . . . . 1005

\section{Personalbereitstellung und Personalfuihrung}

von Prof. Dr. Hugo Kossbiel, Hamburg . . . . . . . . . . . . . . . . . 1007

A. Allgemeine personalwirtschaftliche Bezüge . . . . . . . . . . . . 1011

B. Die Personalbereitstellung . . . . . . . . . . . . . 1013

I. Grundlagen . . . . . . . . . . . . . . . . 1013

II. Problembereiche der Personalbereitstellung . . . . . . . . . . 1016

1. Der Personalbedarf . . . . . . . . . . . . . . 1016

a) Determinanten des Personalbedarfs . . . . . . . . . 1016

b) Ermittlung des Personalbedarfs . . . . . . . . . . . . . 1017 
V. Bilanzierung der Passiva . . . . . . . . . . . . . 795

1. Eigenkapital .................. 795

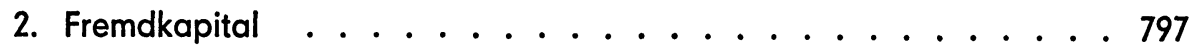

VI. Gewinn- und Verlustrechnung _. . . . . . . . . 800

1. Aufbau der Gewinn- und Verlustrechnung . . . . . . . . 800

2. Ausweis von Aufwendungen und Erträgen . . . . . . . . 805

3. Rücklagebewegung . . . . . . . . . . . . . . 814

VIl. KonzernabschluB . . . . . . . . . . . . . . . . 815

1. Konsolidierungsbereich . . . . . . . . . . . 815

2. Kapitalkonsolidierung .............. 820

3. Schuldenkonsolidierung . . . . . . . . . 822

4. Bewertung der Vermögensgegenstände . . . . . . . . 823

5. Konzernrücklagen und Konzerngewinn . . . . . . . . 827

6. Konzern-Gewinn- und Verlustrechnung . . . . . . . . 828

7. Konzernbilanz . . . . . . . . . . . . . 832

VIII. Analyse des Jahresabschlusses . . . . . . . . . . . . 835

1. Stille Rücklagen . . . . . . . . . . . . 835

2. Liquiditätsanalyse . . . . . . . . . . 838

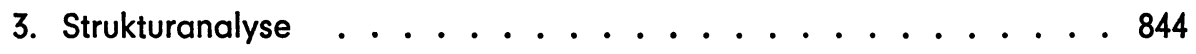

4. Erfolgsanalyse . . . . . . . . . . . . . 849

IX. Bilanztheorie . . . . . . . . . . . . . 857

Literaturverzeichnis . . . . . . . . . . . . . 875

Stichwortverzeichnis . . . . . . . . . . . . . 881

\section{Betriebliches Rechnungswesen}

von Prof. Dr. Wolfgang Kilger, Saarbrücken . . . . . . . . . . 883

1. Aufgaben und Grundbegriffe des betrieblichen Rechnungswesens . . . . 887

11. Aufgaben und Teilgebiete . . . . . . . . . . . 887

12. Abgrenzung zwischen Auszahlung, Beschaffungswert, Aufwand und Kosten . . . . . . . . . . . . . . . . . 891

13. Abgrenzung zwischen Einzahlung, Erlös, Ertrag und Leistung . . . . 899 
2. Die Personalausstattung . . . . . . . . . . . . . 1023

a) Aktions- und Entscheidungsparameter der Personalausstattung . 1023

b) Formale Erfassung der Personalausstattung . . . . . . . 1027

3. Der Personaleinsatz . . . . . . . . . . . . . . . . 1029

a) Alternativen des Personaleinsatzes . . . . . . . . . . . 1029

b) Formale Erfassung des Personaleinsatzes . . . . . . . . . 1034

III. Lösungsansätze zur Personalbereitstellung . . . . . . . . . . 1035

1. Konzeptionelle Probleme der Personalplanung . . . . . . . . 1035

a) Strukturprobleme . . . . . . . . . . . . . . 1035

b) Abstimmungsprobleme . . . . . . . . . . . . . 1036

c) Informationsprobleme . . . . . . . . . . . . . . 1042

2. Modelle zur Planung der Personalbereitstellung . . . . . . . 1043

a) Klassifikation der Modelle . . . . . . . . . . . . . . . 1043

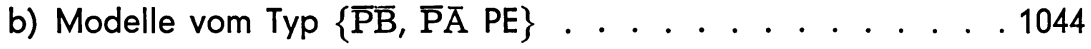

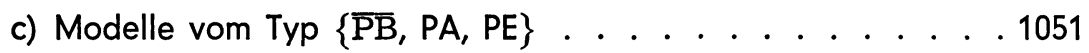

d) Modelle vom $\operatorname{Typ}\{\mathrm{PB}, \overline{\mathrm{P}} \overline{\mathrm{A}}, \mathrm{PE}\} \ldots . . .1063$

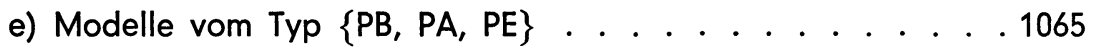

C. Die Personalführung . . . . . . . . . . . . . . . 1075

I. Grundlagen . . . . . . . . . . . . . . . 1075

1. Begriff und Problembereiche der Personalführung . . . . . . 1075

2. Relevanz der sogenannten Führungstheorien . . . . . . . . 1077

II. Problembereiche und Lösungsansätze _ . . . . . . . . . . . 1082

1. Aufgabenidentifikation und Aufgabenklassifikation . . . . . 1082

a) Exkurs: Notwendigkeit der Personalführung . . . . . . . . 1082

b) Aufgabenidentifikation . . . . . . . . . . . 1085

c) Aufgabenklassifikation . . . . . . . . . . . . 1087 
2. Entwicklung von Beeinflussungsstrategien . . . . . . . . 1090

a) Exkurs: Das Legitimationsproblem . . . . . . . . . . 1090

b) Beeinflussungsstrategien auf individual-psychologischer Grundlage

(1) Ein Handlungsmodell . . . . . . . . . . . . . 1093

(2) Lenkung des Verhaltens . . . . . . . . . . . 1095

(a) Grundlegung: Alternativenspektrum und Motivationsaktualisierung . . . . . . . . 1095

(b) Beeinflussung über Ergebnis-Valenzen . . . . . . 1107

(c) Beeinflussung über Ergebnis-Wahrscheinlichkeiten . . 1117

(d) Relevanz individueller Konflikte . . . . . . . 1120

(3) Kontrolle des Verhaltens . . . . . . . . . . . . 1124

c) Beeinflussungsstrategien auf sozialwissenschaftlicher Grundlage . . . . . . . . . . . . . . 1127

(1) Grundlegung: Interaktionelle Beziehungen . . . . . . 1127

(2) Beeinflussung von Gruppenstrukturen und Gruppenprozessen . . . . . . . . . . . 1129

(3) Handhabung sozialer Konflikte . . . . . . . . . 1134

3. Konzeptionen der Personalführung . . . . . . . . . . 1141

a) Führungsstile . . . . . . . . . . . . . . . . . 1142

(1) Differenzierung und Kennzeichnung von Führungsstilen . . 1142

(2) Beurteilung von Führungsstilen . . . . . . . . . . 1149

b) "Führungsprinzipien" (Management-Techniken) . . . . . . 1154

c) Das Kontingenzmodell der Führung . . . . . . . . . . 1158

Literaturverzeichnis . . . . . . . . . . . . . 1165

Stichwortverzeichnis . . . . . . . . . . . . . . . 1173

Gesamt-Stichwortverzeichnis . . . . . . . . . . . . . 1177 